\title{
Impact of the pump wavelength in THz emissions by two-color femtosecond laser filaments in air
}

\author{
A. Nguyen $^{1}$, P. González de Alaiza Martínez ${ }^{1,2}$, J. Déchard ${ }^{1}$, I. Thiele ${ }^{2}$, I. Babushkin ${ }^{3,4}$, S. Skupin $^{2}$, and \\ L. Bergé ${ }^{1}$ \\ 1. CEA-DAM, DIF, 91297 Arpajon, France \\ 2. Univ. Bordeaux - CNRS - CEA, Centre Lasers Intenses et Applications, UMR 5107, 33405 Talence, France \\ 3. Institute of Quantum Optics, Universität Hannover, Welfengarten 1 30167, Hanover, Germany \\ 4. Max Born Institute, Max Born Str. 2a, 12489 Berlin, Germany
}

Laser filamentation is actively studied for its rich variety of applications, from supercontinuum generation to lightning control [1]. In air, femtosecond filaments result from the self-focusing of ultrashort light pulses that couple to their own plasma channel and stay self-guided upon extended paths at high intensity levels. Driven by strong nonlinearities, these optical structures are able to promote broadband THz-to-far-infrared radiation when using laser fields composed of two colors, e.g., a fundamental and its second harmonic [2].

We first present a numerical model unifying the major sources known for $\mathrm{THz}$ emissions by laser-plasma interactions, namely, ponderomotive forces [3], photocurrents induced by air ionization [4] and optical rectification by four-wave mixing [5]. We show that, although photocurrents provide the major conversion mechanism in air-based filaments, the $\mathrm{THz}$ spectrum accumulated over several centimeters results in fact from the combined influence of both plasma and Kerr nonlinearities. The Kerr response of air mainly affects the $\mathrm{THz}$ range through the spectral distortions of the laser pulse. In particular, the delayed nonlinearity attached to Raman scattering of air molecules changes the pulse spectrum along the propagation, which conditions the photocurrents and locally decreases the $\mathrm{THz}$ yield.
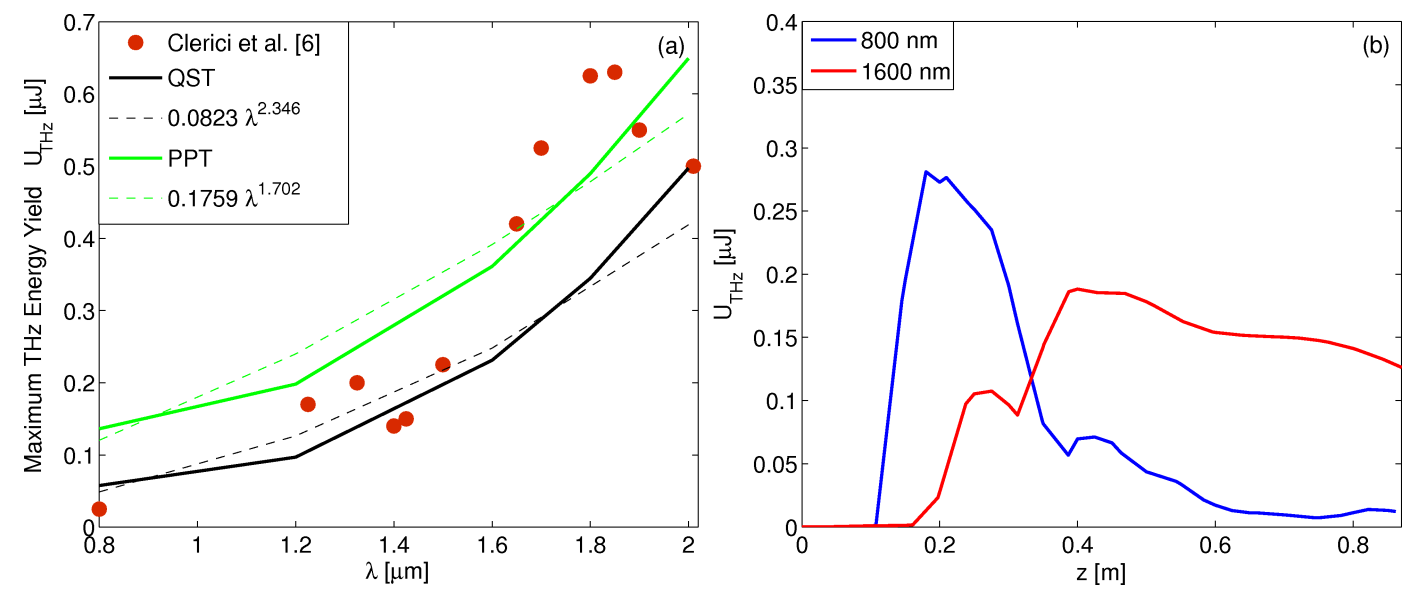

Figure 1 (a) 3D simulation results of THz yields computed from a unidirectional pulse propagator for focused two-color 60-fs Gaussian pulses with a quasi-static ionization model (black curves) or the Perelomov-Popov-Terent'ev ionization rate (green curves) for air molecules. Maximum THz energy yield $(v \leq 80 \mathrm{THz})$ as a function of the pump wavelength and corresponding scaling curves in $\lambda^{\alpha}$ (dashed curves). The red dots recall the experimental data points of [6]. (b) THz energy in a 80-THz frequency window of two-color 40-fs filaments simulated for 800-nm (blue curves) and 1600-nm (red curves) pump pulses.

Next, by means of the local current theory and comprehensive 3D numerical simulations, we confirm the prominent growth in the THz energy yield reported when the wavelength of the fundamental pulse, $\lambda$, is increased [6]. However, we demonstrate that the THz energy variations cannot be quantitatively formulated through a simple power law in $\lambda^{\alpha}$ due to the rapid change in the relative phase between the two colors [7]. Scalings in $\lambda^{\alpha}$ with $\alpha \sim 2$ have been extracted in focused propagation geometries, which reproduce experimental measurements of $\mathrm{THz}$ pulse energies [Figure 1(a)]. In contrast, similar scalings cannot be reached in a long-range filamentation geometry that features much lower plasma densities at longer wavelengths, as illustrated by Figure 1(b).

\section{References}

[1] L. Bergé et al., "Ultrashort filaments of light in weakly ionized, optically transparent media," Rep. Prog. Phys. 70, 1633 (2007).

[2] J. F. Daigle et al., "Remote THz generation from two-color filamentation: long distance dependence," Opt. Express 20, 6825 (2012).

[3] C. D'Amico et al., "Forward THz radiation emission by femtosecond filamentation in gases: theory and experiment," New J. Phys. 10, 013015 (2007).

[4] I. Babushkin et al., "Tailoring terahertz radiation by controling tunnel photoionization events in gases," New J. Phys. 13, 123029 (2011).

[5] D. J. Cook and R. M. Hochstrasser, "Intense terahertz pulses by four-wave rectification in air," Opt. Lett. 25, 1210 (2000).

[6] M. Clerici et al., "Wavelength scaling of terahertz generation by gas ionization," Phys. Rev. Lett. 110, 253901 (2013).

[7] A. Nguyen et al., "Spectral dynamics of THz pulses generated by two-color laser filaments in air: the role of Kerr nonlinearities and pump wavelength," to appear in Opt. Express (2017), arXiv:1611.05851v2. 